\title{
10 IDR: A PROPOSAL FOR MANAGING INTER-ORGANIZATIONAL BUSINESS PROCESSES BY USING WEB-SERVICES ORIENTED ARCHITECTURES
}

Franco, R.D., Ortiz Bas A., Anaya, V. and Lario, F.C. Research Centre on Production Management and Engineering Polytechnic University of Valencia

Cami de Vera S /N. Building 7D, SPAIN

Tel. (+34) 96.387.96.80/Fax: (+34) 96.387.76.89 \{dfrancol aortiz I vanaya | fclario\}@cigip.upv.es

Collaborative Networked Organizations are gaining more attention from research bodies and academics as an emergent form of conducting business.

This emergence is mostly based on the recognition of the improvements being achieved by the deployment of global business processes between their members. In addition, business requirements are demanding higher interoperability levels in order to be able to present quick response before environmental events quickly.

Services Oriented Architectures are becoming the right choice in enabling such interoperable environments. They allow members be as independent as possible by managing their own policies, practices or supporting systems and applications. But, by contrast, additional capabilities in terms of being able to provide and/or consume electronic services to/from third parties will be also required.

The IDR project will deploy a CNO platform upon a Service Oriented Architecture by deploying global business processes as web services orchestration.

\section{MOTIVATION}

Collaborative Networked Organizations (CNOs) are gaining more attention from research bodies and academics as an emergent form of conducting business. They tie together two or more companies that had understood the benefits of conducting winwin partnerships coming from such collaborative environments (Camarinha-Matos and Afsarmanesh, 2001; Ortiz et al, 2003).

From this point of view, within such a collaborative environment and although every organisation keeps its independency and control over its own activities, there 
is always an interdependence that gives it the vision of a unique entity, but certainly increases interoperability requirements.

New technologies, mainly those related to the Internet are allowing the deployment of global distributed business processes (DBP), facilitating the interoperability of the information systems in which they are supported.

This work is included in the efforts within the IDR project which is an undergoing initiative founded by the Office for Science and Technology of the Valencian Government. Its main objective is the deployment of a platform that being based on web services orchestration, allows the re-engineering, deployment, execution and monitoring of DBP in a network of companies surrounding an SME in the automotive sector.

The paper is structured as follows: section two analyses CNO adaptability requirements; section three explains how service oriented architectures are becoming such required technology where distributed business processes can be deployed and executed. Finally, we depict how such topics will be developed in IDR.

\section{INTEROPERABILITY IN COLLABORATIVE NETWORKED ORGANIZATIONS}

\subsection{CNO interoperability requirements}

A CNO must respond or react to several events that take place both inside and outside its boundary. Considering the response capability to face the different types of events, it is possible to determine three types of CNO (see Figure 1):

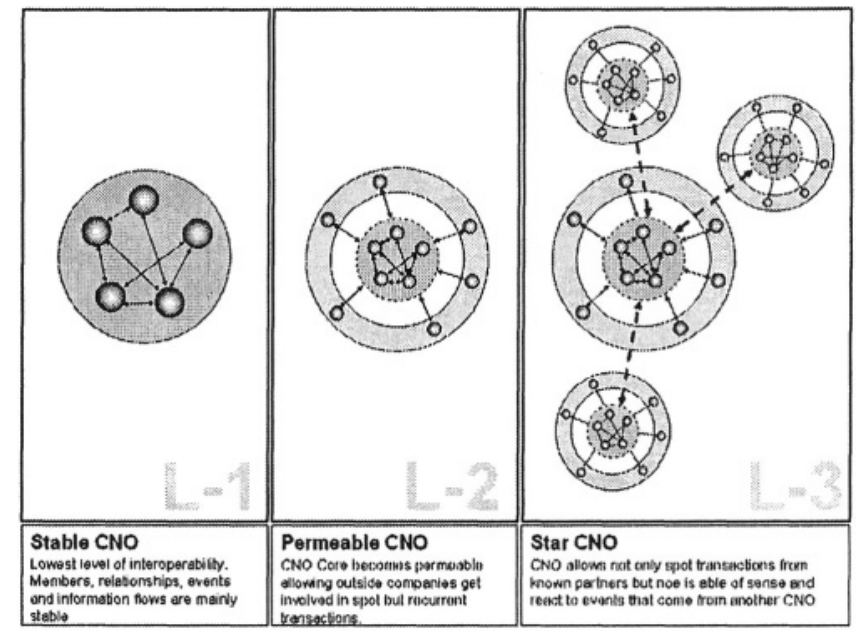

Figure 1: A CNO and its interoperability levels

- Stable CNO: this CNO is composed by relatively stable set of partners who conduct more or less foreseen transactions. No external event can be sensed and there are no mechanisms to properly react to it. For instance, no external order or Request for Quotation (RFQ) can be processed. 
- Permeable CNO: this CNO is intended to be more open than the previous one by allowing third parties to join and leave the network as needed, being able to conduct sporadic transactions through the platform's use.

- Star CNO: when a network is able to effectively react to external events coming from unexpected and unknown sources, for instance an RFQ coming from a distinct CNO.

\subsection{Enablers of a $\mathrm{CNO}$}

Companies looking for higher profitability margins, efficiency and production speed, are no longer focusing into cost reduction or volume increase as the way to achieve them but into bundling a set of services that by itself adds value to the product (in the sense of Smith and Fingar, 2003).

This does not only require providing services or products as usually. At the same time, the nature of offers by using information technologies is being transformed to a set of publicity available services that can be accessed by other interested parties. So named e-Services practices can be defined as the provision of services over electronic networks (Rust and Kannan, 2002). The concept encompasses any kind of electronic relationship being conducted between two organizations.

In doing so, it is necessary that companies evolve from one organizational model that has been mainly based on managing business process in a local way, and where the other members of the value systems are merely "external entities", to a vision within which processes are conceived in a global and mutually engineered way, understanding that major improvements can only be achieved by deploying a set of common policies, practices, rules and platforms supported on widely recognized and standardized technological initiatives (Hagel and Brown, 2002).

In addition, from the technological dimension point of view, Internet-related technologies, with Web Services and its related standards, are leading such an evolution, enabling interoperable environments by creating a new way of architecturally describing systems or applications (Britton, 2001).

\section{SERVICE ORIENTED ARCHITECTURES MEET CNO REQUIREMENTS}

\subsection{Introduction}

Managing distributed business processes in a $\mathrm{CNO}$ requires the achievement of the highest possible degree of independence between its nodes by managing their own policies, practices or supporting systems and applications. But, by contrast, additional capabilities in terms of being able to provide and/or consume electronic services to/from third parties will be also required. Thus, a CNO platform must allow deploying global business processes as a composition of web services invocations (activities).

We consider that a single web service interface definition can be used to wrap a single activity, a sub-process or a full process, mapping it to the proper process model. Doing so, each CNO member will take in charge to execute some activity 
contributing to the overall process but hiding behind the web service definition how, internally, the activity is carried out.

\subsection{Web Services extended}

The web services model is based on the existence of a repository within which different business domains expose (publish or offer) services that can be located and used by other business domains.

Although this is a relatively simple model, the potentiality comes given by its underlying technologies. The use of standards protocols and other Internet's standards guarantees that the services publication and consume between heterogeneous information systems is possible.

According to Kraft (2002) the web service concept can be extended to consider it as an object web service. Doing so, it is possible to analyse them like objects that have a set of methods that can be accessed by their interfaces (object oriented programming analogy).

Formally, a method of an object web service can be defined as:

where:

$$
\mathrm{m}=(\mathrm{i}, \mathrm{p}, \mathrm{r}(\mathrm{p}), \mathrm{M})
$$

$\mathrm{i}=\mathrm{a}$ unique global identifier for the object (i.e. URN)

$\mathrm{p}=$ is a set of entrance parameters

$\mathrm{r}=$ is a computational function that generates an output from $\mathrm{p}$

$\mathrm{M}=$ meta data that provide complementary information for $\mathrm{m}$

A method of a web service object, represented through its interface, can be acceded with the aim of carrying out any computational calculus. From a set of input parameters, a web service object method could produce and output. This invocation might produce an estate change over the object.

Regarding $\mathrm{p}$ and $\mathrm{r}(\mathrm{p})$ are allocated with types of data of a programming language that, in the case of this definition, accepts XML schemes as a possible codification for such information.

Schematically, a method can be represented as:

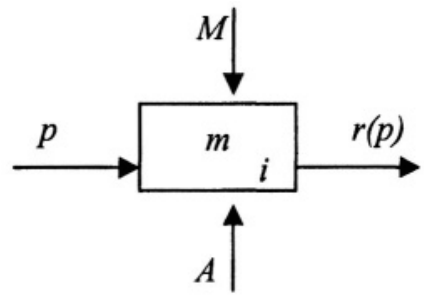

(a)

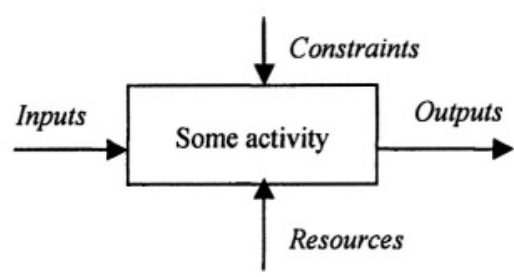

(b)

Figure 2: Representation of a web service method and an IDEF0 activity comparison 


\subsection{Web services as activity wrappers}

In the last point, it was introduced a representation for the web service object methods. In the Error! Reference source not found.2(a) it is possible to see how a method has associated a unique identifier I, which joined to the container's 1 and $\mathrm{b}$ identifiers, allow discovering the web service within the net.

If we also know the interface by which to provide it with $\mathrm{p}$, it is possible to make its invocation (the access always controllers to A allow it) and to obtain the output $r$ (p).

The access controllers' function should not be limited to the application of security control mechanisms. Adopting a higher level of abstraction, it is possible to conceive its function as the one in charge to determine whether the method of invocation is possibly under the current constraints: security, capacity or resources accessibility ones.

We consider that web services objects methods can be used as constructive blocks of the distributed business processes, thus each of them as attended as an activity itself.

\subsection{Mapping business process and web services models}

From the analysis of Figure 2 it is possible to establish an analogy between the representation of both a web service method and a process' activity. Basically, both enable that, under determined constraints (A and $\mathrm{C}$, respectively), to transform a set of inputs (p and I) in an output $(\mathrm{r}(\mathrm{p}))$ and $\mathrm{O}$, respectively.

We consider that a business process can be represented as a set of activities that get executed following a determined sequence. Assuming that, it is possible to make the previous analogy is valid: the composition of invocations to web services methods can become a representation of a business process or sub process over an specific domain.

The next figure presents the model of a business process and the correspondence with one web services composition.
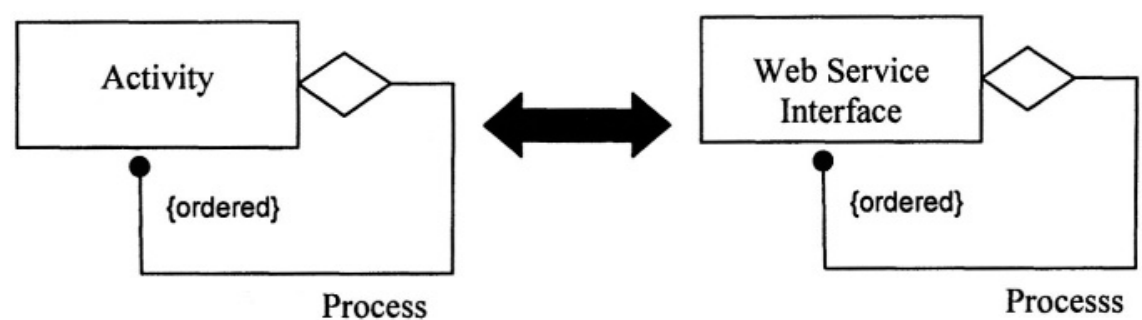

Figure 3: Analogy between business and technological models

At any decomposition level, any modelled activity or sub-process can be associated with a web service interface specification by specifying its interface in terms of inputs, outputs and resources. 


\subsection{Service Oriented Architectures: closing the gap}

Although the attempts to interconnect two or more companies come from mid 80's, there currently is a high expectation in industry about results that can be achieved by using service oriented architectures. But, what is the main change that has happened in order to be so optimistic about this new wave? We consider that the key points are mainly relays on:

- The capability of closing the gap existing between the modelling and the operational phases of the business process engineering, because we are able of modelling them with Business Process Modelling Notation (BPMN), for instance, and getting one executable version of the process by using BPEL4WS.

- The emerging web-services technologies (SOAP, WSDL, UDDI and BPEL4WS mainly) allow different levels of abstraction for business processes modelling and representation. Then, any $\mathrm{CNO}$ member in charge of executing some assigned activity of the process may locally explode it (preserving the public interface) by using their own BPEL4WS model as well.

- Also, always that one activity interface is preserved executors can be switched (Mowshowitz, 1999) depending on conditions under which process is being executed or criteria to be met.

Next, we will present the IDR model which is mainly based on these principles and technologies.

\section{IDR PROJECT: DYNAMIC INTEGRATION IN NETWORKED ENTERPRISES}

The IDR Project's goal/main objective is to create a service-oriented platform that will enable distributed business process management by orchestrating web services within a network of enterprises in the automotive sector, in order to create/improve the Collaborative Planning Business Process performance.

It's closely related to a just finished EU funded project named V-CHAIN. This project deployed a virtual enterprise infrastructure in an automotive supply chain. The scope was limited to the relationship Original Equipment Manufacturing - First Tier Suppliers. IDR has been conceived as an extension of V-CHAIN trying to span their scope in order to include second tier suppliers, as well.

Then, the Core $\mathrm{CNO}$ is composed of a set of the second and third tiers suppliers and additionally also V-CHAIN B2B integration server will provide valuable information like production plans, availability, constrains, stocks or shipping notes, for instance.

In order to reach such required interoperability levels, the IDR framework has been structured has follows (see Figure 5):

- We have defined a web services portfolio within which all active web services definitions that may be used to compose a business process will be stored. There will be three kind of services: sub-contracted with third 
parties (outside of the $\mathrm{CNO}$ ), internally deployed (owned by the core members) and additionally those services that the $\mathrm{CNO}$ will able to provide to third parties.

- Another core component is the process repository which will store business process definitions that later will be used by the process engine to launch instances inside the network. Three kinds of process will be stored: Common set (core processes that can be reused to compose higher level processes; a set of standardised industry-wide processes and, the third one, those business processes that are specific of our business domain.

- Based on such business process definitions, instances of them may be launched by any member of the $\mathrm{CNO}$ by using the BP engine.

- Additionally, all information and control flows will be supported by a Business Object Document definition stored in a XML Schema repository. They will be used as inputs, outputs or meta-data for activities executions.

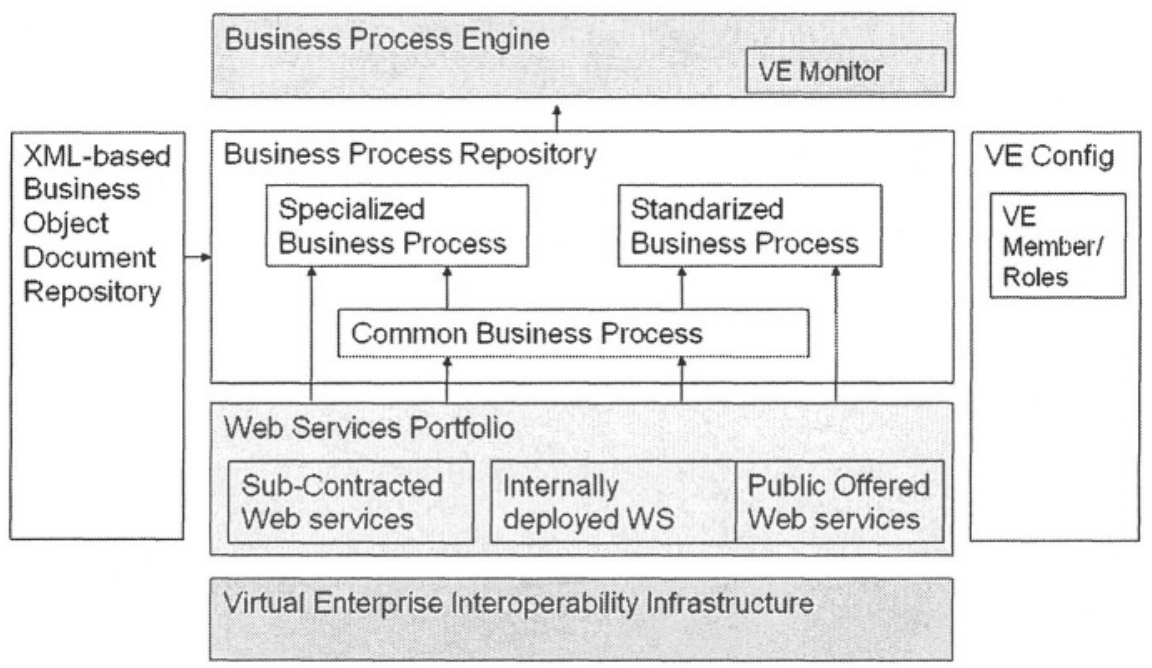

Figure 5: The IDR Platform

From an architectural point of view, tools to be developed include at least (Linthicum, 2004):

- A graphic tool that enables BP design based on BPEL4WS

- A BPM Engine

- A BPM interface (Monitoring process' execution mainly)

- A configuration tool for the CNO

- Metrics: it is expected to release a tool that enables metrics definition and monitoring. 


\section{CONCLUSIONS}

In this work, we have tried to depict how enterprises are facing new challenges in reconfiguring themselves when looking for flexibility augmentation and adaptability.

Internet is enabling Service Oriented Architectures deployment supporting them not only with a set of standards technologies like SOAP, UDDI, WSDL or XML but also with a new modelling languages like BPMN and BPEL4WS that are helping to close the gap existing between engineering and executing phases of distributed business processes management.

The case presented here, the IDR project, is an example that shows how Small and Medium Enterprises (SME) are also embracing such technologies in order to be able to take part in CNO participations or creation.

\section{Acknowledgments}

The IDR (Dynamic Integration in Networked Enterprises) project is an undergoing project founded by Valencia's local government Generalitat Valenciana by means of its Office for Science and Technology.

Part of this work also is supported by the Polytechnic University of Valencia by a Research Grant Program for Predoctoral Fellows.

\section{REFERENCES}

1. Britton, C. (2001). IT Architectures and Middleware: Strategies for Building Large, Integrated Systems. Addison-Wesley.

2. Camarinha-Matos, L. and Afsarmanesh, H. (2001). Dynamic Virtual Organizations, or Not So Dynamic? BASYS Proceedings - Knowledge and technology integration in production and services, Kluwer Academic Publishers, ISBN 1-4020-7211-2, pp. 111-124, Sept 2002.

3. Fingar, P. and Smith, H. (2003). Business Process Management: The third wave. Meghan-Kiffer Press, Tampa, Florida

4. Hagel, J. and Brown, J.S. (2002). Orchestrating Business Processes - Harnessing the value of web services technology. Working Paper available at www.johnhagel.com

5. Kraft, R. (2002). A Model for Network Services on the Web. International Conference on Internet Computing 2002: 536-541

6. Mowshowitz, A. (1999). 'The switching principle in virtual organization', Virtual Organization Net eJOV, vol. 1, no. 1, pp. 6-17

7. Ortiz, A., Franco R.D. y Alba, M. "V-CHAIN: Migrating from Extended to Virtual Enterprise within an Automotive Supply Chain". PROVE'03 Proceedings. Processes and Foundations for Virtual Organizations.

8. Rust, R.T. and Kannan, P.K. (2002) E-Service: New Directions in Theory and Practice. ME Sharpe, Armonk, New York. 